\title{
Approaching Inpatient Glycemic Control Using Six Sigma Methodology
}

\author{
Rodhan Khthir, MD, CPHQ, CCD \\ Associate professor of medicine, University of North Dakota, USA \\ Endocrinologist at Sanford Health, Bismarck, ND, USA \\ *Corresponding author: Rodhan Khthir, MD, CPHQ, CCD; rkhthir@yahoo.com
}

Received 11 March 2021;

Accepted 28 March 2021;

Published 10 April 2021

\begin{abstract}
Process variation affects almost all healthcare processes. Wide fluctuation of blood glucose values is very common in hospitalized patients and may impact the outcome of care in negative way. The purpose of this article is to illustrate how to study the process variation using Six Sigma approach and how to us it teaching healthcare quality.
\end{abstract}

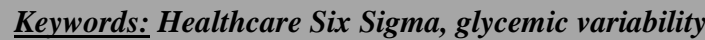

\section{Introduction}

To Err is Human: Building a Safer Health System ${ }^{[1]}$ is a report issued by the U.S. Institute of Medicine (IOM) in November 1999 and has resulted in increasing awareness regarding high medical errors in healthcare industry. The report, which was based on an analysis of adverse health outcomes by a variety of organisations, concluded that between 44,000 and 98,000 people die each year as a result of preventable medical errors. Since its publication; there has been a significant enthusiasm to improve patient safety and to improve healthcare outcome. As a result of that, we have witnessed the introduction of many new innovations and reengineered processes. In spite of that medical error rate remains high, and possibly higher as shown by a more recent medical error analysis [2]

In other industries, any measurement falling outside of industry standards is regarded as a defect. This is how quality is defined, at least in companies that have adopted the Six Sigma approach which is a statistical approach for quality improvement. Processes that operate with "six sigma quality" over the short term are assumed to produce long-term defect levels below 3.4 defects per million opportunities (DPMO) ${ }^{[3]}$. Six Sigma's implicit goal is to improve all processes, but not to the 3.4 DPMO level necessarily. Its main philosophy it to reduce process variation to minimum level using a well-defined statistical approach. Many large companies use Six Sigma methodology to reduce the defect rate to its lowest possible value. The Six Sigma attempts to reduce the number of defects to below 3.4 per million opportunities; industries such as aviation target and achieve an even lower defect rate (less than 1 defect per 2 million opportunities).

Simply, the Six Sigma concept is a statistical approach to improving the quality and performance of a specific process by focusing on the "Critical to Quality Step" as identified by the "Voice of the Customer". It aims to maintain the mean result within a target range (i.e., between upper and lower specification limits) and focuses on reducing the variation in the outcome to the lowest possible level. The variation in outcome is usually measured as the standard deviation around the mean (i.e., Sigma). The Six Sigma method aims to fit six standard deviations around the mean without crossing the lower or upper specification targets. This process yields high performance and high potential ${ }^{[3]}$.

Healthcare processes have usually high defect rate and wide variations (low sigma level). Six Sigma approach can be used in healthcare to improve specific processes using the same methodology used by other industries. The purpose of this analysis is to illustrate how to use Six Sigma approach in healthcare processes to decrease variation, using inpatient glycemic control as an example.

Introducing this approach in medical education can help medical students, residents and other professionals to approach healthcare quality and patient safety in standardized way and can allow them to use recent advances in medical technology and artificial intelligence to achieve better healthcare outcomes.

Another purpose of this article is to illustrate how to study the process variation using Six Sigma approach and how to use it in teaching and approaching healthcare quality. Education on process variation using six sigma methodology is a valuable way to teach the new generation of physicians the statistical approach to quality.

\section{Methods}

Analysis of 9609 glucose data point done in non-ICU units from 2015 was done as part of the baseline data collected for of a 
quality improvement project that aimed to decrease glucose variation in non-ICU adults patients. These data were part of a quality improvement project that aimed to decrease glucose fluctuation across all medical units at a tertiary care center. It was agreed that the "industry standard" for glucose level in hospitalized non-critically ill patients should fall between $100 \mathrm{mg} / \mathrm{dl}$ and 180 $\mathrm{mg} / \mathrm{dl}{ }^{[4]}$.

Using Six Sigma Methodology, the mean, standard deviation, Defect Per Million Opportunity (DPMO), sigma level and Process Performance indices were calculated. Statistical analysis was done using QI Macros 2011 software.

\section{Results}

Plotting glucose data against frequency was done to visualize the data distribution (Figure 1). Adding specification limits: $100 \mathrm{mg} / \mathrm{dl}$ (lower specification limit) and $180 \mathrm{mg} / \mathrm{dl}$ (upper specification limit), provided a visual estimation of process performance. The mean glucose value in the sample was $167.8 \mathrm{mg} / \mathrm{dl}$ with standard deviation of 69.1 . As a process with a target of $100 \mathrm{mg} / \mathrm{dl}$ to 180 $\mathrm{mg} / \mathrm{dl}$, the glycemic control has a very high defect rate of $46.7 \%$ or Defect Per Million Opportunity (DPMO) of 467,478 ((only half of all glucose measurements fell within the target range). The Sigma level was 1.58. The Process performance (Pp) was 0.19 .

The glycemic control data analysis showed high defect rate, and high variation. Sigma level of 1.58 reflects poor process performance and provides great opportunity for improvement.

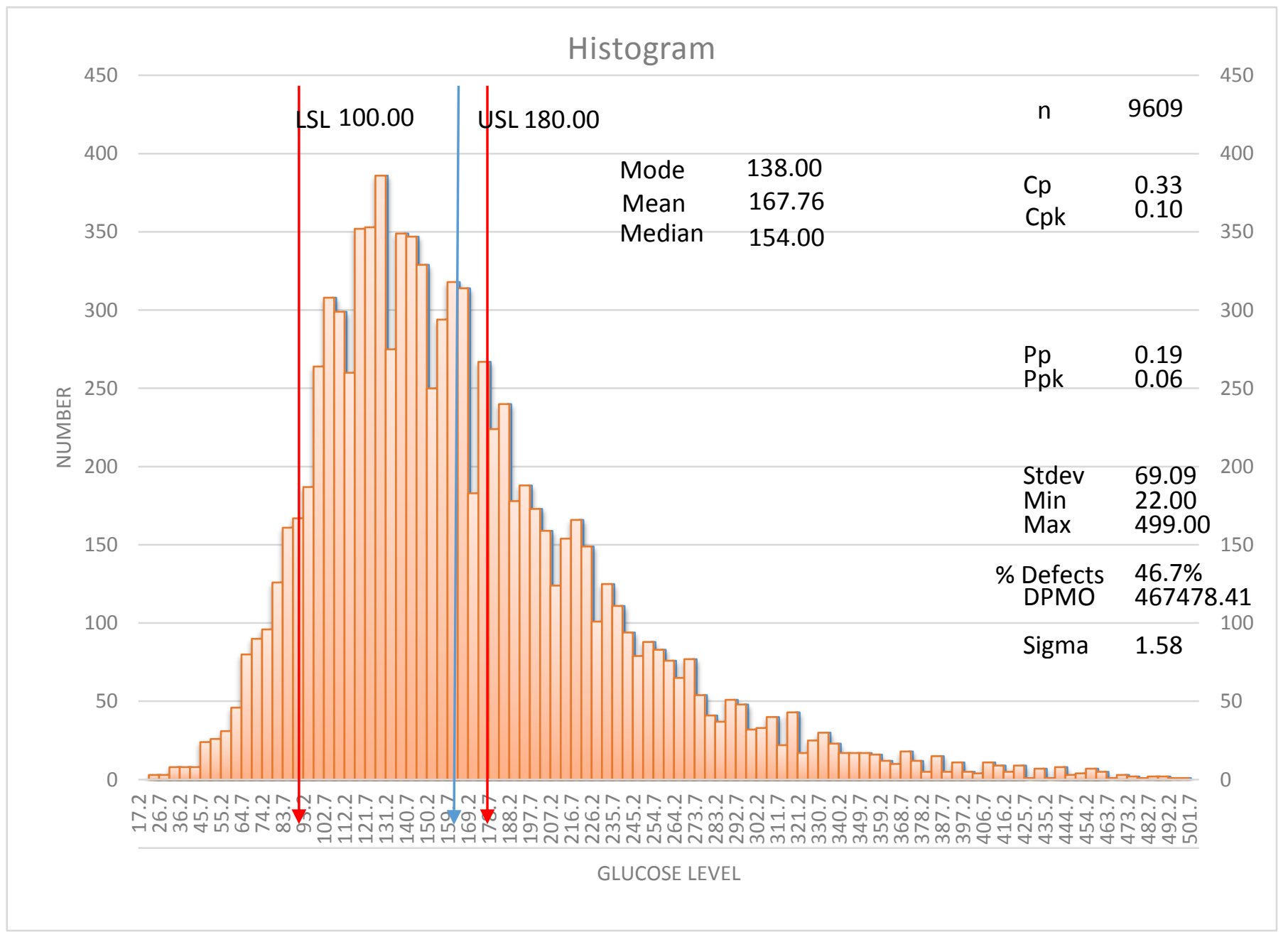

Figure 1: Glycemic control with measurement of Mean, Standard deviation (Stdev). Defect rate (Defect\%), Defect per million opportunity (DPMO), Sigma level (Sigma), Process Performance (Pp), Process performance Index (PpK), Process Capability (Cp) and Process Capability index (Cpk)

\section{Discussion}

Many large companies use Six Sigma methodology to reduce the defect rate to its lowest possible value. The Six Sigma attempts to reduce the number of defects to below 3.4 per million opportunities; industries such as aviation target and achieve an even lower defect rate (less than 1 defect per 2 million opportunities). Simply, the Six Sigma concept is a statistical approach to improving the quality and performance of a specific process by focusing on the "Critical to Quality Step" as identified by the "Voice of the Customer". It aims to maintain the mean result within a target range (i.e., between upper and lower specification limits) and focuses on reducing the variation in the outcome to the lowest possible level. The variation in outcome (the killer in any industrial process) is usually measured as the standard deviation around the mean (i.e., Sigma). The Six Sigma method aims to fit six standard deviations around the mean without crossing the lower or upper specification targets. This process yields high performance and high potential. In our example of a mean glucose level of $167.8 \mathrm{mg} / \mathrm{dl}$ and a standard deviation of $69.1 \mathrm{mg} / \mathrm{dl}$, we can calculate the Process Performance or Process Potential $(\mathrm{Pp})$ for our data $\left(\mathrm{Pp}=(\mathrm{USL}-\mathrm{LSL}) / 6^{*} \mathrm{~s}\right.$. where $\mathrm{s}$ is the standard deviation $)$.

Using Six Sigma language to describe our data, we find that the number of defects is 467478.41 per million opportunities (DPMO), with a Sigma level of 1.58. The current performance of this process has a process potential $(\mathrm{Pp})$ of only 0.19 , when ideally, 
the Pp should be $>1$ (indicative of a process for which 6 standard deviations fit on either side of the mean between the upper and lower specification limits).

For continuous measurements such as glucose level, we usually consider the mean level as a performance indicator. Even in the outpatient setting, we measure $\mathrm{HbA1c}$ level to represent glycemic control. A good mean glucose level with high variability is not physiologically equivalent to a good mean glucose with low variability ${ }^{[5]}$. The former is associated with a higher defect rate (low sigma level), higher chance of hypoglycemia, and higher oxidative stress in association with severe hyperglycemia or a sudden rise in glucose level. Better performance measures are available to determine the performance of inpatient glycemic control. All of these methods focus on measuring glycemic variability as an independent predictor of clinical outcome. GV can be defined more simply as the degree to which a patient's blood glucose level fluctuates between high (peaks) and low (nadir) levels. Standard deviation, glycemic range and the Mean Amplitude of Glycemic Excursions (MAGE) are common metrics used to assess GV. The MAGE is calculated by measuring the arithmetic mean of the difference between consecutive peaks and nadirs, provided that the difference is greater than the SD around the mean glucose values.

Decreasing the mean glucose level to $140 \mathrm{mg} / \mathrm{dl}$ and decreasing the standard deviation to $5 \mathrm{mg} / \mathrm{dl}$ will yield a Six Sigma process with a process potential of $>1$. Is this achievable in practice? As an endocrinologist, I believe that decreasing the mean glucose level to $140 \mathrm{mg} / \mathrm{dl}$ using standardized protocols and clinical pathways would be easy to achieve. On the other hand, decreasing glucose variability as measured by the standard deviation in hospitalized patients is an extremely difficult goal. However, levels of approximately 20 or 30 or even $40 \mathrm{mg} / \mathrm{dl}$ are likely attainable and could improve the Sigma level and process potential, resulting in a lower defect rate and reduced glucose variability $(\mathrm{GV})$. The use of continuous glucose monitoring devices, insulin pump and diabetes management software in the inpatient settings can all help improving glycemic variability and reduce number of defects. The advances in the use of artificial intelligence can introduce a much higher level of care if coupled for example with sensor augmented insulin pumps designed specifically for the inpatient settings. Such approach can decrease defect rate and can improve process performance significantly.

Fifteen years after the publication of the "To Err is Human" ${ }^{, 1]}$ report, the incidence of medical errors and adverse events remain on the rise. The famous Institute of Medicine (IOM) report indicated that between 44,000 and 98,000 patients die in US hospitals every year because of medical errors. For an industry such as aviation or companies such as Boeing or GE, the response to a much smaller error rate would be nothing less than to radically re-engineer and re-design systems and processes. Our response in healthcare was to improve in some areas with the help of initiatives by agencies such as the Institute for Healthcare Improvement (IHA), the Joint Commission, the IOM and the Agency for Healthcare Research and Quality (AHQR). These initiatives and innovations helped to improve the safety of healthcare in specific areas such as congestive heart failure, myocardial infarction, bronchial asthma, and surgical site infection, but in contrast to other industries, we did not make radical changes at the system level nor make efforts to change the way that we approach healthcare processes. As a result, the Office of the Inspector General has reported that 180,000 Medicare patients die from hospital mishaps per year ${ }^{[6]}$. In 2013, a research study published in the Journal of Patient Safety estimated between 210,000 and 400,000 deaths from avoidable medical errors occur annually ${ }^{[2]}$.

\section{Conclusion}

In summary, this example illustrates the need for innovation in the way that we evaluate healthcare processes and outcomes and in the way that we teach medicine. For inpatient glycemic control, regarding outlier glucose readings as defects and considering glucose variability in terms of Sigma level and process potential will help us to make progress towards the nearly defect-free industrial standards achieved in certain fields.

Our medical students and residents encounter a large number of defects every day (e.g., hypoglycemia or severe hyperglycemia) and often understand the root cause of the defect (i.e., not administering insulin on time, administering too much or too little insulin, and not adjusting for renal function or food intake) without much reaction most of the time.

I believe that we must radically change our approach to healthcare delivery, as well as our perception and attitude toward medical errors and adverse events. We need to introduce the concept of reducing process variability and defects by teaching medical, nursing and pharmacy students the industrial approach to quality, using concepts such as lean methodology, statistical process control and Six Sigma. Teaching medical quality through reducing process variation approach at the very early stages of the "process of making a physician" will help to create a new generation of physicians with completely different attitude and perception of medical errors and defects.

\section{Ethics approval and consent to participate}

Not applicable as the De- identified glucose data was obtained as part of the regular ongoing quarterly quality auditing.

\section{List of abbreviations}

Institute of Medicine (IOM)

Defects per million opportunities (DPMO)

Process performance (Pp)

USL: Upper Specification limit

LSL: Lower Specification Limit

Mean Amplitude of Glycemic Excursions (MAGE)

Standard Deviation (SD)

Glucose variability (GV)

Institute for Healthcare Improvement (IHA)

The Agency for Healthcare Research and Quality (AHQR)

\section{Data Availability}

De- identified glucose data was obtained as part of the regular quarterly quality auditing by laboratory department and Diabetes committee at St. Mary's Medical Center, Huntington, West Virginia, USA

\section{Conflicts of Interest}

The author declares that there is no conflict of interest regarding the publication of this paper.

\section{Funding Statement}

Not applicable 


\section{References}

[1] Institute of Medicine (IOM). To Err is Human: Building a Safer Health System. Washington, DC: National Academy Press 1999.

[2] James JT. A new, evidence-based estimate of patient harms associated with hospital care. J Patient Saf 2013;9:122-8.

[3] Roderick A, Matthew J. Maio, Mohamed B. Nawaz, Govindarajan Ramu and Daniel J. Zrymiak. The Certified Six Sigma Green Belt Handbook. ASQ Quality Press 2007.
[4] Standars of medical care in Diabetes-2021. Diabetes Care. January 2021. Volume 44. Supplement 1

[5] Gorst C, Kwok CS, Aslam S, Buchan I, Kontopantelis E, Myint PK, Heatlie G, Loke Y, Rutter MK, Mamas MA Long-term Glycemic Variability and Risk of Adverse Outcomes: A Systematic Review and Meta-analysis. Diabetes Care. 2015 Dec;38(12):2354-69

[6] Office of Inspector General reports: Medical Mistakes Plague Medicare Patientshttp://oig.hhs.gov/newsroom/newsreleases/2010/plague.asp 